\title{
Downstream Targets of FOXM1: CEP55 and HELLS are Cancer Progression Markers of Head and Neck Squamous Cell Carcinoma
}

\author{
Ahmad Waseem ${ }^{a}$, Muhammad Ali ${ }^{a}$, Edward W. Odell ${ }^{b}$, Farida Fortune ${ }^{a}$ and Muy-Teck Teh ${ }^{a_{1} *}$ \\ ${ }^{a}$ Centre for Clinical and Diagnostic Oral Sciences, Institute of Dentistry, Barts and The London School \\ of Medicine and Dentistry, Queen Mary University of London, Turner Street, London E1 2AD, \\ England, United Kingdom.
}

${ }^{\mathrm{b}}$ Head and Neck Oncology Group, King's College London Dental Institute, Guy's Hospital Campus, London SE1 9RT, United Kingdom.

\section{${ }^{*}$ Corresponding Author:}

Dr. Muy-Teck Teh

Centre for Clinical \& Diagnostic Oral Sciences

Barts \& the London School of Medicine \& Dentistry

4, Newark Street, London E1 2AT.

United Kingdom.

Tel: +44 (0) 2078827140

Fax: $+44(0) 2078827137$

Email:m.t.teh@qmul.ac.uk

Short Title: CEP55 and HELLS are biomarkers for HNSCC progression

\section{Keywords}

Oral carcinoma, FOXM1, CEP55, HELLS, LSH, PASG, SMARCA6, head and neck, oncogene, diagnostic biomarkers, HNSCC, oral cancer, digital densitometry, early detection, bioinformatics, microarray, immunohistochemistry, qPCR, real-time quantitative PCR.

\begin{abstract}
Abbreviations
HNSCC, head and neck squamous cell carcinoma; FOXM1, forkhead box M1; CEP55, centrosomal protein 55; HELLS, lymphoid specific helicase; qPCR, absolute real time reverse transcription quantitative polymerase chain reaction; NHOK, primary normal human oral keratinocytes; LnMet, lymph node metastasis; FFPE, formalin-fixed paraffin embedded.
\end{abstract}




\section{ABSTRACT}

We recently showed that upregulation of a key oncogene FOXM1 precedes head and neck squamous cell carcinoma (HNSCC) malignancy. Furthermore, we also identified a centrosomal protein CEP55 and a DNA helicase/putative stem cell marker HELLS, which are both downstream targets of FOXM1. In this study, we have investigated the expression profiles of CEP55 and HELLS using immunohistochemistry and quantified by digital densitometry in a tissue panel (20 samples) consisting of normal oral mucosa, dysplasias, HNSCC and lymph node metastasis (LnMet) samples. Furthermore, we corroborated our findings using absolute real-time PCR (qPCR) on a panel of 12 primary normal human oral keratinocytes, 5 dysplasia and 10 HNSCC cell lines. Finally, we validated our study using bioinformatics microarray analysis on an independent HNSCC patient cohort (4 normal and 16 tumours). In normal oral mucosa, CEP55 protein was detected at very low level within the upper differentiated layers. In contrast, CEP55 was highly expressed in oral dysplasia whereas only moderate expression was detected in HNSCC and LnMet. Low level of HELLS expression was detected in the basal cell layer of the normal oral mucosa, moderate level was seen in dysplasia and high levels in both HNSCC and LnMet. These expression patterns were consistent with both qPCR data from the cell line panel and microarray data analysis of TNM-stage defined HNSCC samples confirming the progressive expression pattern of CEP55 and HELLS. To our knowledge, this is the first pilot study demonstrating that both CEP55 and HELLS mRNA and protein expression positively correlate with pre-malignancy and HNSCC progression. This study provides strong evidence that CEP55 and HELLS may be used in conjunction with FOXM1 as a biomarker set for early cancer detection and indicators of malignant conversion and progression. 


\section{INTRODUCTION}

Head and neck squamous cell carcinoma (HNSCC) is the $6^{\text {th }}$ most common cancer worldwide and it has higher death rates than cancers of the colon, breast, vulva or melanoma. ${ }^{1}$ In the UK, over 4700 new cases are reported annually. Its impact on health services, the patients, their families and society in general is financially crippling because of the cost of treatment and the effect on the patients' cosmetic appearance, speech and eating. In recent years, there has been evidence of an increasing rate of HNSCC incidence in several European countries probably due to the changing trends of alcohol and tobacco consumption and exposure, especially in females. ${ }^{2}$ Because of late tumour detection coupled with high recurrence rate after treatment, the 5-year survival of patients remains poor (average 50\%, depending on clinical stage at diagnosis). ${ }^{1}$ This supports the need for better screening and diagnostic interventions to improve early detection of HNSCC.

The human transcription factor FOXM1 gene consists of 10 exons located on the 12p13.3 chromosomal band (telomeric position). Two exons, named exons Va and Vlla, also referred to as exon A1 (or rat exon 6) and A2 respectively, are alternatively spliced. ${ }^{3,4}$ FOXM1 has a role in cell cycle, DNA repair and maintenance of genomic stability. ${ }^{5}$ We were the first to establish a link between FOXM1 and oncogenesis when we showed that FOXM1 was upregulated in basal cell carcinoma, ${ }^{3}$ the most common type of cancer in Caucasians worldwide. Subsequent studies of FOXM1 in many different solid human cancer types confirmed its pivotal role in oncogenesis. ${ }^{5,6}$ We have recently reported that transcriptional upregulation of FOXM1 precedes malignancy in a number of solid human cancer types including oral, oesophagus, lung, breast, kidney, bladder and uterus. ${ }^{7}$ It has been shown that FOXM1 interacts with the human papilloma virus (HPV) 16 E7 protein $^{8}$ and that HPV16 E6/E7 has been implicated in HNSCC. ${ }^{9}$ It is likely that HPV may play a role in HPV-positive HNSCC through FOXM1 activation. However, it has been shown that the frequency of HNSCCs that have integrated HPV and express high levels E7 is only in the region of $4-16 \%{ }^{10,11}$ 
Teh et al. - CEP55 \& HELLS are Biomarkers for HNSCC Progression

We have recently shown that aberrant upregulation of FOXM1 serves as a first oncogenic hit by destabilising the genome and subsequent activation of downstream targets which regulates cytokinesis (CEP55, centrosomal protein $55 \mathrm{kDa})^{12-14}$ and epigenetic/stem-cell factor re-programming (HELLS, helicase lymphoid specific) ${ }^{15-18}$ leading to malignant transformation (anchorage-independent growth) of a premalignant oral keratinocyte line (SVpgC2a). ${ }^{7}$ However, the protein expression profiles of CEP55 and HELLS during HNSCC progression have not been described. In this study, we have characterised the expression patterns of CEP55 and HELLS using immunohistochemistry and digital densitometry to quantify their protein levels in an HNSCC progression tissue panel consisting of normal oral mucosa, dysplasias, HNSCC and lymph node metastasis (LnMet). We have complemented these findings using absolute real-time reverse transcription PCR (qPCR) on a panel of primary normal human oral keratinocytes, dysplasia and HNSCC cell lines. The data suggest that CEP55 and HELLS in combination with FOXM1 are early markers of cancer detection and they are also indicators of malignant conversion and progression.

\section{MATERIALS \& METHODS}

\section{Clinical Samples}

The use of human tissue was approved by the relevant Research Ethics Committees at each institution. The present study involved 20 patients' formalin-fixed paraffin embedded (FFPE) archival tissues as previously described. ${ }^{7}$

\section{Immunohistochemistry and digital pixel densitometry}

FOXM1 was immunostained with a rabbit polyclonal antibody (K-19; Santa Cruz Biotechnology, California, USA) and mouse monoclonal antibodies for both CEP55 (AB57537-100; Abcam plc, Cambridge, UK) and HELLS (anti-LSH H-4 SC-46665; Santa Cruz) on paraffin sections as described. ${ }^{3,}$ 7, 19 The reaction product was visualised with diaminobenzidine as a chromogenic 
Teh et al. - CEP55 \& HELLS are Biomarkers for HNSCC Progression

substrate in FFPE tissue sections. As described previously, ${ }^{7,20,21}$ digital pixel densitometry was performed using Adobe Photoshop CS3 (Adobe Systems Incorporated, USA) utilising the Colour Range tool for colour-based quantification or Lasso tool for manual selection of epithelial mucosa area and subsequent pixel area readout from the Histogram data chart. Whilst we have also used the ImageJ program for pixel densitometry and obtained similar results, Photoshop was preferred for its accuracy, reproducibility and ease of use.

\section{Cell culture}

Primary normal human oral keratinocytes (12 different NHOK primary cultures) were cultured in a modified version of Keratinocyte SFM (K-SFM, \#17005-034 Gibco, Invitrogen) medium ${ }^{22}$ containing $0.09 \mathrm{mM} \mathrm{CaCl}$. K-SFM was supplemented with $25 \mu \mathrm{g} / \mathrm{ml}$ bovine pituitary extract, $0.2 \mathrm{ng} / \mathrm{ml}$ EGF (\#37000-015 Gibco, Invitrogen), and 1\% penicillin/streptomycin. Oral premalignant cell lines: OKF6/T, ${ }^{23}$ POE9n, ${ }^{22}$ DOK, ${ }^{24}$ D $19,{ }^{7}$ D20, ${ }^{7}$ and primary HNSCC derived cell lines: SCC,${ }^{25}$ SCC $15,{ }^{25}$ $\mathrm{SCC} 25,{ }^{25} \mathrm{SqCC} / \mathrm{Y} 1,{ }^{26} \mathrm{UK} 1,{ }^{27} \mathrm{VB} 6,{ }^{27} \mathrm{CaLH} 2,{ }^{27} \mathrm{CaDec} 12,{ }^{27} 5 \mathrm{PT},{ }^{27} \mathrm{H} 357^{27}$ were cultured as described previously. ${ }^{7}$

\section{Real-time absolute quantitative RT-PCR}

Poly-A+ mRNA extraction, reverse transcription and real-time absolute quantitative PCR (qPCR) protocols are MIQE compliant ${ }^{28}$ and were performed as described previously ${ }^{7}$ using a LightCycler LC480 instrument (Roche Diagnostic). Primer sequences for FOXM1B, CEP55, HELLS and two stable reference genes (POLR2A and YAP1) were described previously. ${ }^{7}$ Statistical analysis was performed using the GraphPad InStat software (V2.04a, GraphPad Software, San Diego, CA) for Student's t-test analysis.

\section{Bioinformatics analyses of microarray data}

FOXM1, CEP55 and HELLS endogenous mRNA levels were analysed using published microarray data in a study using normal oral mucosa and a panel of TNM-staged defined HNSCC tissues ${ }^{29}$. The microarray dataset (GDS1584) was obtained from the NCBI's Gene Expression Omnibus (GEO) 
Teh et al. - CEP55 \& HELLS are Biomarkers for HNSCC Progression

database. The Affymetrix gene reference identification for FOXM1, CEP55 and HELLS are 202580_x_at, 218542_at and 220085_at, respectively. Gene expression levels were statistically analysed using Student's t-test in Microsoft Excel software.

\section{RESULTS}

\section{Protein Expression patterns of CEP55 and HELLS during HNSCC progression}

In order to validate our previous finding that CEP55 and HELLS are targets of FOXM1, we investigated the CEP55 (Fig. 1) and HELLS (Fig. 2) protein expression levels by immunohistochemistry on a panel of 5 normal human oral mucosa, 5 oral dysplasia, 5 primary HNSCC and 5 LnMet FFPE archival tissues. In normal oral mucosa, very low level of CEP55 immunostaining was detected in the middle/upper suprabasal layers (Fig. 1A,B - Normal). As the staining was restricted only to the keratinised layers we believe that this may be non-specific staining. In contrast, intense but heterogeneous nuclear and cytoplasmic CEP55 expression was detected in oral dysplasia within the basaloid suprabasal layers (Fig. 1A,B - Dysplasia). Moderately upregulated level of cytoplasmic CEP55 protein was detected in HNSCC tissues (Fig. 1A,B - HNSCC). In LnMet, CEP55 exhibits a heterogeneous expression pattern with higher cytoplasmis and nuclear protein levels detected along the periphery of tumour mass (Fig. 1A,B - LnMet). Digital densitometry was used to quantify the levels of CEP55 expression where 6-10 digital images were captured and quantified from each tissue specimen. Individual data points for each tissue specimen were plotted as a scatter plot (Fig. 1C) and Box-and-Whisker plot (Fig. 1D) to illustrate the distribution pattern of CEP55 protein expression across the tissue panel. Mean \pm SEM analysis plot (Fig. 1E) showed that CEP55 was significantly upregulated in dysplasia and HNSCC. The upregulation of CEP55 in LnMet was not significant due to tissue heterogeneity. 
Teh et al. - CEP55 \& HELLS are Biomarkers for HNSCC Progression

In normal oral mucosa, HELLS nuclear immunostaining was detected strictly within the basal layer of the oral mucosa epithelium (Fig. 2A,B - Normal). In oral dysplasia, HELLS nuclear expression was moderately upregulated and found throughout the basaloid layers (Fig. 2A,B - Dysplasia). High level of nuclear HELLS protein was detected in HNSCC tumour masses (Fig. 2A,B - HNSCC). In LnMet, HELLS exhibits a heterogeneous nuclear expression pattern (Fig. 2A,B - LnMet). Individual data points for each tissue specimen were plotted as a scatter plot (Fig. 2C) and Box-and-Whisker plot (Fig. 2D) as described above for CE55 expression. The mean expression \pm SEM analysis plot (Fig. 2E) showed that HELLS was significantly upregulated in all three disease tissues with a progressive trend.

\section{Correlation of CEP55 and HELLS mRNA expression with FOXM1}

It has been previously established that FOXM1B shows a progressive expression pattern during HNSCC progression. ${ }^{7}$ Since CEP55 and HELLS are downstream targets of FOXM1B, we hypothesise that their expression patterns should show a positive correlation with FOXM1B. To test this hypothesis, we measured the mRNA expression levels of FOXM1B, CEP55 and HELLS in a panel of 12 primary normal human oral keratinocytes, 5 dysplasia (OKF6/T, POE9n, DOK, D19 and D20) and 10 primary HNSCC derived keratinocyte cell lines (SCC9, SCC15, SCC25, SqCC/Y1, UK1, VB6, CaLH2, CaDec12, 5PT and H357) using absolute qPCR. Both CEP55 (Fig. 3A) and HELLS (Fig. 3B) showed good expression correlation with FOXM1B across the panel of cell lines used.

\section{Bioinformatics microarray analysis of CEP55 and HELLS expression}

To further validate the above findings, we performed bioinformatics analysis on an independent HNSCC patient cohort comparing 4 normal oral mucosa and 16 TNM-stage defined HNSCC samples. $^{29}$ The gene expression data of CEP55 and HELLS were extracted from the microarray dataset (GDS1584) using the NCBI's Gene Expression Omnibus online research tool as described previously. ${ }^{7}$ The expression patterns of FOXM1, CEP55 and HELLS all showed similar bell-shaped 
Teh et al. - CEP55 \& HELLS are Biomarkers for HNSCC Progression

profile across the different TNM tumour stages (Fig. 1C) with peak expression at early-mid tumour stages (T1N2bM0 to T4N2bM0) and decrease expression found in more advance tumour stages (T4N2M0 and T4N3M0). Statistical analysis showed that FOXM1 and CEP55 were significantly upregulated in the HNSCC $(n=16)$ compared to normal tissues $(n=4)$ (Fig. 3D). The upregulation of HELLS expression in HNSCC was not statistically significant due to a greater heterogeneous gene expression within the different HNSCC tumour stages.

\section{DISCUSSION}

It is now well established that FOXM1 is a major oncogene in myriad of human cancers including HNSCC. $^{7,30}$ In our previous study, we identified CEP55 and HELLS as two downstream targets of FOXM1. ${ }^{7}$ Herein, we have provided the first protein expression evidence that both CEP55 and HELLS are differentially expressed during tumour progression from dysplasia to HNSCC and LnMet in human oral tissues. Both their expression patterns correlated well with that of FOXM1 expression profile across a cell line panel containing normal oral keratinocytes, dysplasia and HNSCC derived cells. The progressive pattern of gene expression of CEP55 and HELLS was further validated using bioinformatics microarray data analysis on an independent cohort of HNSCC study.

CEP55 is a microtubule-bundling protein that localizes to the mitotic spindle during prometaphase and metaphase and to the spindle midzone and the midbody during anaphase and is required for the completion of cytokinesis. ${ }^{12-14}$ Consistent with CEP55 being a direct transcriptional target of FOXM1, ${ }^{7}$ both CEP55 ${ }^{12}$ and FOXM1 ${ }^{31}$ share organ-specific expression in the testis and thymus of normal adult humans. CEP55 is upregulated in hepatocellular carcinoma and its overexpression induces anchorage-independent growth, enhanced cell growth at low serum levels and induction of tumourigenesis in nude mice. ${ }^{32}$ Another study showed that CEP55 was overexpressed in lung cancer 
and is associated with cell migration and invasion. ${ }^{33}$ Consistent with a role in malignancy, similar to the expression of FOXM1, ${ }^{7}$ we found that CEP55 expression is significantly upregulated in both oral dysplasia and HNSCC tissues. Interestingly, a study involving 3 independent breast cancer patient cohorts of microarray datasets containing a total of 699 patients revealed that CEP55 and FOXM1 are amongst the signature prognostic markers which predict poor breast cancer outcome ${ }^{34}$ Another study showed that FOXM1 and CEP55 were ranked $3^{\text {rd }}$ and $43^{\text {rd }}$, respectively, most significant predictor genes for poor clinical outcome in 6 different cancer types. ${ }^{35}$ Consistent with being a potential cancer biomarker, CEP55 has been shown to act as a novel breast carcinoma-associated antigen ${ }^{36}$ which has important implications in cancer diagnostic and/or therapeutic interventions.

Whilst we have previously demonstrated using chromatin-immunoprecipitation that FOXM1 protein directly transactivated the promoter of CEP55 to induce gene expression, we find no evidence of a FOXM1-binding site on the HELLS promoter, despite showing a strong correlation in gene expression between FOXM1 and HELLS. ${ }^{7}$ Our current data also showed significant correlation in both protein and mRNA expression pattern between HELLS and FOXM1 during HNSCC progression. Similar to FOXM1 ${ }^{31}$ and CEP55, ${ }^{12}$ HELLS also showed organ-specific expression in the testis and thymus. ${ }^{37}$ Nevertheless, because only $1 \mathrm{~kb}$ of the HELLS promoter region was analysed, ${ }^{7}$ the FOXM1 binding site(s) could be located beyond the HELLS promoter region tested. Further study involving a larger promoter region of HELLS is required to establish the basis for HELLS induction by FOXM1.

HELLS is a member of the SNF2 (Sucrose Non-Fermenter) family of helicase proteins implicated in mammalian development and cellular proliferation, ${ }^{16,38}$ through chromatin-remodelling and DNA methylation. ${ }^{39-42}$ Whilst deletion of HELLS gene causes developmental retardation, premature ageing and cellular senescence, ${ }^{38,43-45}$ gene modification or upregulation of HELLS has been implicated in various human cancer including leukaemia, ${ }^{37}$ non-small cell lung cancer, ${ }^{46}$ breast cancer, ${ }^{47}$ and melanoma. ${ }^{48}$ HELLS was recently identified as one of the consensus genes expressed in human embryonic stem cells. ${ }^{17}$ Recently, DNA methyltransferase 1 (DNMT1), an interacting partner of 
HELLS, ${ }^{42}$ was shown to maintain progenitor function in self-renewing epidermis ${ }^{49}$ highlighting a role for HELLS in progenitor cell maintenance and renewal. Interestingly, in the present study, HELLS expression was strictly restricted to the basal layer of the normal mucosa epithelium and that upregulation of HELLS was found in the hyperplastic basal and suprabasal layers in oral dysplasia. This pattern of HELLS expression appear highly similar to that of FOXM1 immunostaining in basal cell carcinomas $^{3}$ and in HNSCC. ${ }^{7}$ We therefore speculate that the upregulation of HELLS (and FOXM1) in HNSCC may indicate a deregulation of progenitor cell maintenance leading to hyperproliferation and tumour development.

In summary, this study provided the first evidence that a novel set of proliferation-associated genes FOXM1, CEP55 and HELLS share progressive expression pattern during the progression of HNSCC. The fact that upregulation of these three genes precedes HNSCC malignancy renders them potential cancer biomarkers for early detection of malignant conversion. This pilot study would pave way for future studies involving more tissue samples for validating their diagnostic potential as biomarkers for HNSCC.

\section{ACKNOWLEDGEMENTS}

This study was supported by the Institute of Dentistry, Barts and The London School of Medicine and Dentistry. We thank Professor E. Ken Parkinson (Centre for Clinical \& Diagnostic Oral Science) for providing SCC9, SCC15 and SCC25 cell lines and critical reading of the manuscript. We also thank Professor lan Mackenzie (Centre for Cutaneous Research, Blizard Institute of Cell and Molecular Science) for providing POE9n, DOK, D19, D20, UK1, VB6, CaLH2, CaDec12 and 5PT cell lines.

\section{Conflict of Interest}

None declared. 


\section{REFERENCES}

1. Parkin, D.M., Bray, F., Ferlay, J. \& Pisani, P. Global cancer statistics, 2002. CA Cancer J Clin 2005;55(2):74-108.

2. La Vecchia, C., Lucchini, F., Negri, E. \& Levi, F. Trends in oral cancer mortality in Europe. Oral Oncol 2004;40(4):433-439.

3. Teh, M.T., Wong, S.T., Neill, G.W., Ghali, L.R., Philpott, M.P. \& Quinn, A.G. FOXM1 is a downstream target of Gli1 in basal cell carcinomas. Cancer Res 2002;62(16):4773-4780.

4. Wierstra, I. \& Alves, J. FOXM1, a typical proliferation-associated transcription factor. Biol Chem 2007;388(12):1257-1274.

5. Myatt, S.S. \& Lam, E.W. The emerging roles of forkhead box (Fox) proteins in cancer. Nat Rev Cancer 2007;7(11):847-859.

6. Laoukili, J., Stahl, M. \& Medema, R.H. FoxM1: at the crossroads of ageing and cancer. Biochim Biophys Acta 2007;1775(1):92-102.

7. Gemenetzidis, E., Bose, A., Riaz, A.M., Chaplin, T., Young, B.D., Ali, M. et al. FOXM1 upregulation is an early event in human squamous cell carcinoma and it is enhanced by nicotine during malignant transformation. PLOS ONE 2009;4(3):e4849.

8. Luscher-Firzlaff, J.M., Westendorf, J.M., Zwicker, J., Burkhardt, H., Henriksson, M., Muller, R. et al. Interaction of the fork head domain transcription factor MPP2 with the human papilloma virus 16 E7 protein: enhancement of transformation and transactivation. Oncogene 1999;18(41):56205630.

9. Wiest, T., Schwarz, E., Enders, C., Flechtenmacher, C. \& Bosch, F.X. Involvement of intact HPV16 E6/E7 gene expression in head and neck cancers with unaltered p53 status and perturbed pRb cell cycle control. Oncogene 2002;21(10):1510-1517.

10. Ha, P.K., Pai, S.I., Westra, W.H., Gillison, M.L., Tong, B.C., Sidransky, D. et al. Real-time quantitative PCR demonstrates low prevalence of human papillomavirus type 16 in premalignant and malignant lesions of the oral cavity. Clin Cancer Res 2002;8(5):1203-1209.

11. Braakhuis, B.J., Snijders, P.J., Keune, W.J., Meijer, C.J., Ruijter-Schippers, H.J., Leemans, C.R. et al. Genetic patterns in head and neck cancers that contain or lack transcriptionally active human papillomavirus. J Natl Cancer Inst 2004;96(13):998-1006.

12. Fabbro, M., Zhou, B.B., Takahashi, M., Sarcevic, B., Lal, P., Graham, M.E. et al. Cdk1/Erk2- and Plk1-dependent phosphorylation of a centrosome protein, Cep55, is required for its recruitment to midbody and cytokinesis. Dev Cell 2005;9(4):477-488.

13. Martinez-Garay, I., Rustom, A., Gerdes, H.H. \& Kutsche, K. The novel centrosomal associated protein CEP55 is present in the spindle midzone and the midbody. Genomics 2006;87(2):243-253.

14. Zhao, W.M., Seki, A. \& Fang, G. Cep55, a microtubule-bundling protein, associates with centralspindlin to control the midbody integrity and cell abscission during cytokinesis. Mol Biol Cell 2006;17(9):3881-3896.

15. Geiman, T.M., Durum, S.K. \& Muegge, K. Characterization of gene expression, genomic structure, and chromosomal localization of Hells (Lsh). Genomics 1998;54(3):477-483.

16. Geiman, T.M. \& Muegge, K. Lsh, an SNF2/helicase family member, is required for proliferation of mature T lymphocytes. Proc Natl Acad Sci U S A 2000;97(9):4772-4777.

17. Assou, S., Le Carrour, T., Tondeur, S., Strom, S., Gabelle, A., Marty, S. et al. A meta-analysis of human embryonic stem cells transcriptome integrated into a web-based expression atlas. Stem Cells 2007;25(4):961-973.

18. McGraw, S., Vigneault, C. \& Sirard, M.A. Temporal expression of factors involved in chromatin remodeling and in gene regulation during early bovine in vitro embryo development. Reproduction 2007;133(3):597-608.

19. Ghali, L., Wong, S.T., Green, J., Tidman, N. \& Quinn, A.G. Gli1 protein is expressed in basal cell carcinomas, outer root sheath keratinocytes and a subpopulation of mesenchymal cells in normal human skin. J Invest Dermatol 1999;113(4):595-599. 
20. Teh, M.T., Blaydon, D., Ghali, L.R., Edmunds, S., Pantazi, E., Barnes, M.R. et al. Role for WNT16B in human epidermal keratinocyte proliferation and differentiation. $J$ Cell Sci 2007;120(2):330-339.

21. Lalli, A., Tilakaratne, W.M., Ariyawardana, A., Fitchett, C., Leigh, I.M., Hagi-Pavli, E. et al. An altered keratinocyte phenotype in oral submucous fibrosis: correlation of keratin K17 expression with disease severity. J Oral Pathol Med 2008;37(4):211-220.

22. Rheinwald, J.G., Hahn, W.C., Ramsey, M.R., Wu, J.Y., Guo, Z., Tsao, H. et al. A two-stage, p16(INK4A)- and p53-dependent keratinocyte senescence mechanism that limits replicative potential independent of telomere status. Mol Cell Biol 2002;22(14):5157-5172.

23. Dickson, M.A., Hahn, W.C., Ino, Y., Ronfard, V., Wu, J.Y., Weinberg, R.A. et al. Human keratinocytes that express hTERT and also bypass a p16(INK4a)- enforced mechanism that limits life span become immortal yet retain normal growth and differentiation characteristics. Mol Cell Biol 2000;20(4):1436-1447.

24. Chang, S.E., Foster, S., Betts, D. \& Marnock, W.E. DOK, a cell line established from human dysplastic oral mucosa, shows a partially transformed non-malignant phenotype. Int $\mathrm{J}$ Cancer 1992;52(6):896-902.

25. Rheinwald, J.G. \& Beckett, M.A. Tumorigenic keratinocyte lines requiring anchorage and fibroblast support cultures from human squamous cell carcinomas. Cancer Res 1981;41(5):1657-1663.

26. Reiss, M., Pitman, S.W. \& Sartorelli, A.C. Modulation of the terminal differentiation of human squamous carcinoma cells in vitro by all-trans-retinoic acid. J Natl Cancer Inst 1985;74(5):10151023.

27. Locke, M., Heywood, M., Fawell, S. \& Mackenzie, I.C. Retention of intrinsic stem cell hierarchies in carcinoma-derived cell lines. Cancer Res 2005;65(19):8944-8950.

28. Bustin, S.A., Benes, V., Garson, J.A., Hellemans, J., Huggett, J., Kubista, M. et al. The MIQE guidelines: minimum information for publication of quantitative real-time PCR experiments. Clin Chem 2009;55(4):611-622.

29. Toruner, G.A., Ulger, C., Alkan, M., Galante, A.T., Rinaggio, J., Wilk, R. et al. Association between gene expression profile and tumor invasion in oral squamous cell carcinoma. Cancer Genet Cytogenet 2004;154(1):27-35.

30. Myatt, S.S. \& Lam, E.W. Targeting FOXM1. Nat Rev Cancer 2008;8(3):242.

31. Ye, H., Kelly, T.F., Samadani, U., Lim, L., Rubio, S., Overdier, D.G. et al. Hepatocyte nuclear factor 3/fork head homolog 11 is expressed in proliferating epithelial and mesenchymal cells of embryonic and adult tissues. Mol Cell Biol 1997;17(3):1626-1641.

32. Chen, C.H., Lu, P.J., Chen, Y.C., Fu, S.L., Wu, K.J., Tsou, A.P. et al. FLJ10540-elicited cell transformation is through the activation of PI3-kinase/AKT pathway. Oncogene 2007;26(29):42724283.

33. Chen, C.H., Lai, J.M., Chou, T.Y., Chen, C.Y., Su, L.J., Lee, Y.C. et al. VEGFA upregulates FLJ10540 and modulates migration and invasion of lung cancer via PI3K/AKT pathway. PLoS ONE 2009;4(4):e5052.

34. Martin, K.J., Patrick, D.R., Bissell, M.J. \& Fournier, M.V. Prognostic breast cancer signature identified from 3D culture model accurately predicts clinical outcome across independent datasets. PLOS ONE 2008;3(8):e2994.

35. Carter, S.L., Eklund, A.C., Kohane, I.S., Harris, L.N. \& Szallasi, Z. A signature of chromosomal instability inferred from gene expression profiles predicts clinical outcome in multiple human cancers. Nat Genet 2006;38(9):1043-1048.

36. Inoda, S., Hirohashi, Y., Torigoe, T., Nakatsugawa, M., Kiriyama, K., Nakazawa, E. et al. Cep55/c10orf3, a tumor antigen derived from a centrosome residing protein in breast carcinoma. $J$ Immunother 2009;32(5):474-485.

37. Lee, D.W., Zhang, K., Ning, Z.Q., Raabe, E.H., Tintner, S., Wieland, R. et al. Proliferationassociated SNF2-like gene (PASG): a SNF2 family member altered in leukemia. Cancer Res 2000;60(13):3612-3622. 
38. Raabe, E.H., Abdurrahman, L., Behbehani, G. \& Arceci, R.J. An SNF2 factor involved in mammalian development and cellular proliferation. Dev Dyn 2001;221(1):92-105.

39. Dennis, K., Fan, T., Geiman, T., Yan, Q. \& Muegge, K. Lsh, a member of the SNF2 family, is required for genome-wide methylation. Genes Dev 2001;15(22):2940-2944.

40. Yan, Q., Cho, E., Lockett, S. \& Muegge, K. Association of Lsh, a regulator of DNA methylation, with pericentromeric heterochromatin is dependent on intact heterochromatin. Mol Cell Biol 2003;23(23):8416-8428.

41. Yan, Q., Huang, J., Fan, T., Zhu, H. \& Muegge, K. Lsh, a modulator of CpG methylation, is crucial for normal histone methylation. EMBO J 2003;22(19):5154-5162.

42. Myant, K. \& Stancheva, I. LSH cooperates with DNA methyltransferases to repress transcription. Mol Cell Biol 2008;28(1):215-226.

43. Geiman, T.M., Tessarollo, L., Anver, M.R., Kopp, J.B., Ward, J.M. \& Muegge, K. Lsh, a SNF2 family member, is required for normal murine development. Biochim Biophys Acta 2001;1526(2):211-220.

44. Sun, L.Q., Lee, D.W., Zhang, Q., Xiao, W., Raabe, E.H., Meeker, A. et al. Growth retardation and premature aging phenotypes in mice with disruption of the SNF2-like gene, PASG. Genes Dev 2004;18(9):1035-1046.

45. Zhou, R., Han, L., Li, G. \& Tong, T. Senescence delay and repression of p16INK4a by Lsh via recruitment of histone deacetylases in human diploid fibroblasts. Nucleic Acids Res 2009;37(15):5183-5196.

46. Yano, M., Ouchida, M., Shigematsu, H., Tanaka, N., Ichimura, K., Kobayashi, K. et al. Tumorspecific exon creation of the HELLS/SMARCA6 gene in non-small cell lung cancer. Int $J$ Cancer 2004;112(1):8-13.

47. Einbond, L.S., Su, T., Wu, H.A., Friedman, R., Wang, X., Jiang, B. et al. Gene expression analysis of the mechanisms whereby black cohosh inhibits human breast cancer cell growth. Anticancer Res 2007;27(2):697-712.

48. Ryu, B., Kim, D.S., Deluca, A.M. \& Alani, R.M. Comprehensive expression profiling of tumor cell lines identifies molecular signatures of melanoma progression. PLOS ONE 2007;2(7):e594.

49. Sen, G.L., Reuter, J.A., Webster, D.E., Zhu, L. \& Khavari, P.A. DNMT1 maintains progenitor function in self-renewing somatic tissue. Nature 2010;463(7280):563-567.

\section{Figure Legends}

Figure 1. Immunostaining of CEP55 in human normal oral mucosa, dysplasia, HNSCC and lymph node metastasis (LnMet). (A) Two representative images for each tissue category are shown here. (B) Magnified views of selected regions (dotted boxes) in (A) are shown. (C) Scatter plot of digital pixel densitometry data for CEP55 protein immunoreactivity in a panel of 20 oral tissues $(n=5$ in each group) as shown in (A). (D) Box-and-Whisker plot for the same data from (C). (E) Mean analysis of data points from $(C) *(P<0.05)$ and ${ }^{* *}(P<0.01)$ indicate statistically significant elevation of CEP55 protein levels when compared to normal oral mucosa tissues. 
Teh et al. - CEP55 \& HELLS are Biomarkers for HNSCC Progression

Figure 2. Immunostaining of HELLS in human normal oral mucosa, dysplasia, HNSCC and lymph node metastasis (LnMet). (A) Two representative images for each tissue category are shown here. (B) Magnified views of selected regions (dotted boxes) in (A) are shown. (C) Scatter plot of digital pixel densitometry data for HELLS protein immunoreactivity in a panel of 20 oral tissues $(n=5$ in each group) as shown in (A). (D) Box-and-Whisker plot for the same data from (C). (E) Mean analysis of data points from $(C) *(P<0.05)$ and ${ }^{\star * \star}(P<0.001)$ indicate statistically significant elevation of HELLS protein levels when compared to normal oral mucosa tissues.

Figure 3. Gene expression analysis of FOXM1, CEP55 and HELLS in cell line panel and HNSCC tissues. (A) A non-linear regression analysis between CEP55 and FOXM1B mRNA expression levels determined by absolute qPCR across a cell line panel consisting of 12 normal human oral keratinocytes (NHOK), 5 dysplasia and 10 HNSCC cell lines. (B) A non-linear regression analysis between HELLS and FOXM1B mRNA expression levels across the same panel of cell lines used in (A). (C) Bioinformatics analysis of FOXM1, CEP55 and HELLS gene expression from a microarray dataset involving 4 normal oral mucosa and 16 TNM-stage defined HNSCC tissues (GDS1584 ${ }^{29}$ ). (D) Mean analysis of FOXM1, CEP55 and HELLS gene expression using the same dataset from (C) comparing the normal $(n=4)$ and HNSCC $(n=16)$ samples. ${ }^{*}(P<0.05)$ and ${ }^{*}(P<0.01)$ indicate statistically significant gene upregulation in HNSCC when compared to normal oral mucosa tissues. 


\section{Figure 1}
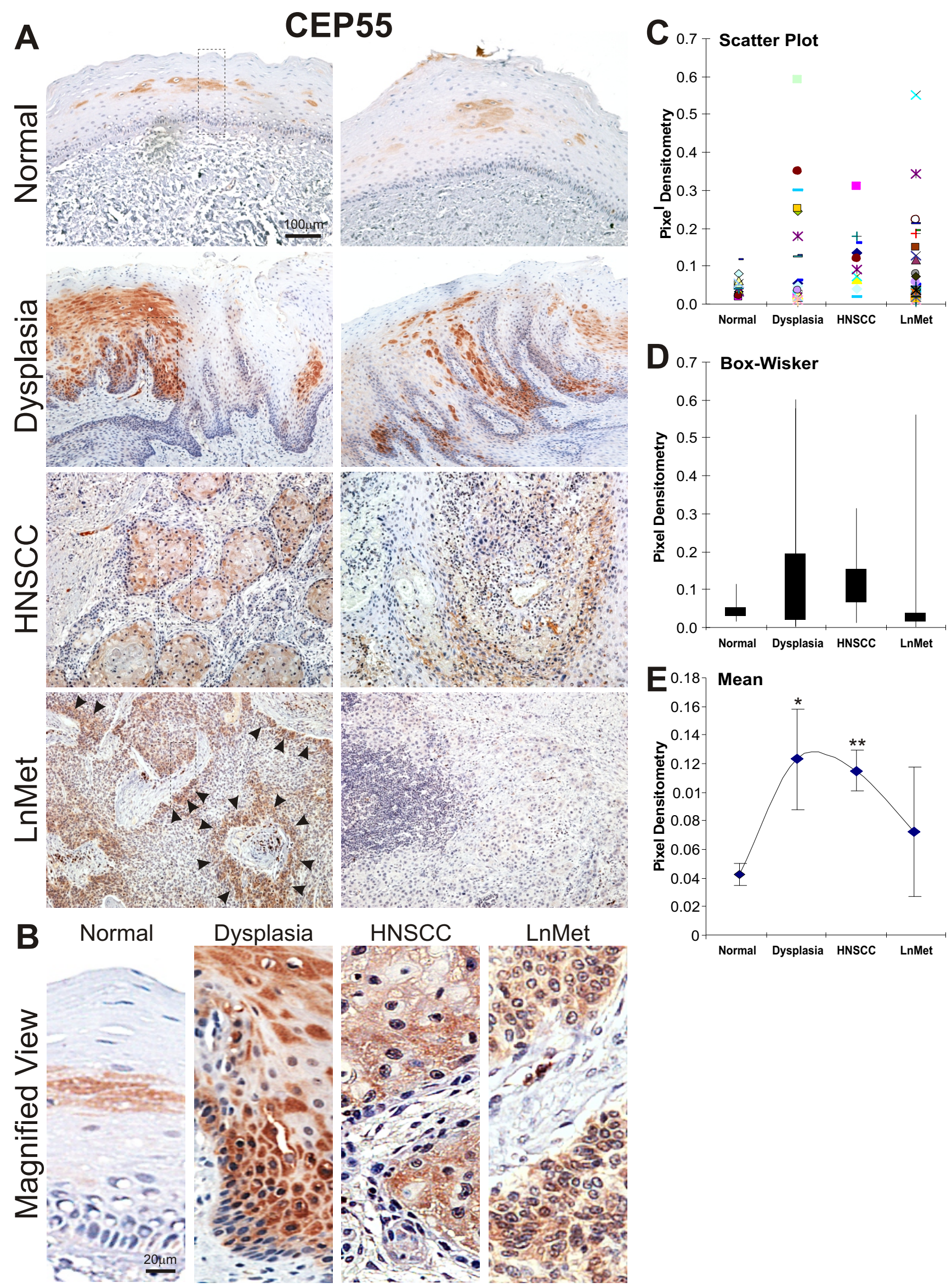


\section{Figure 2}
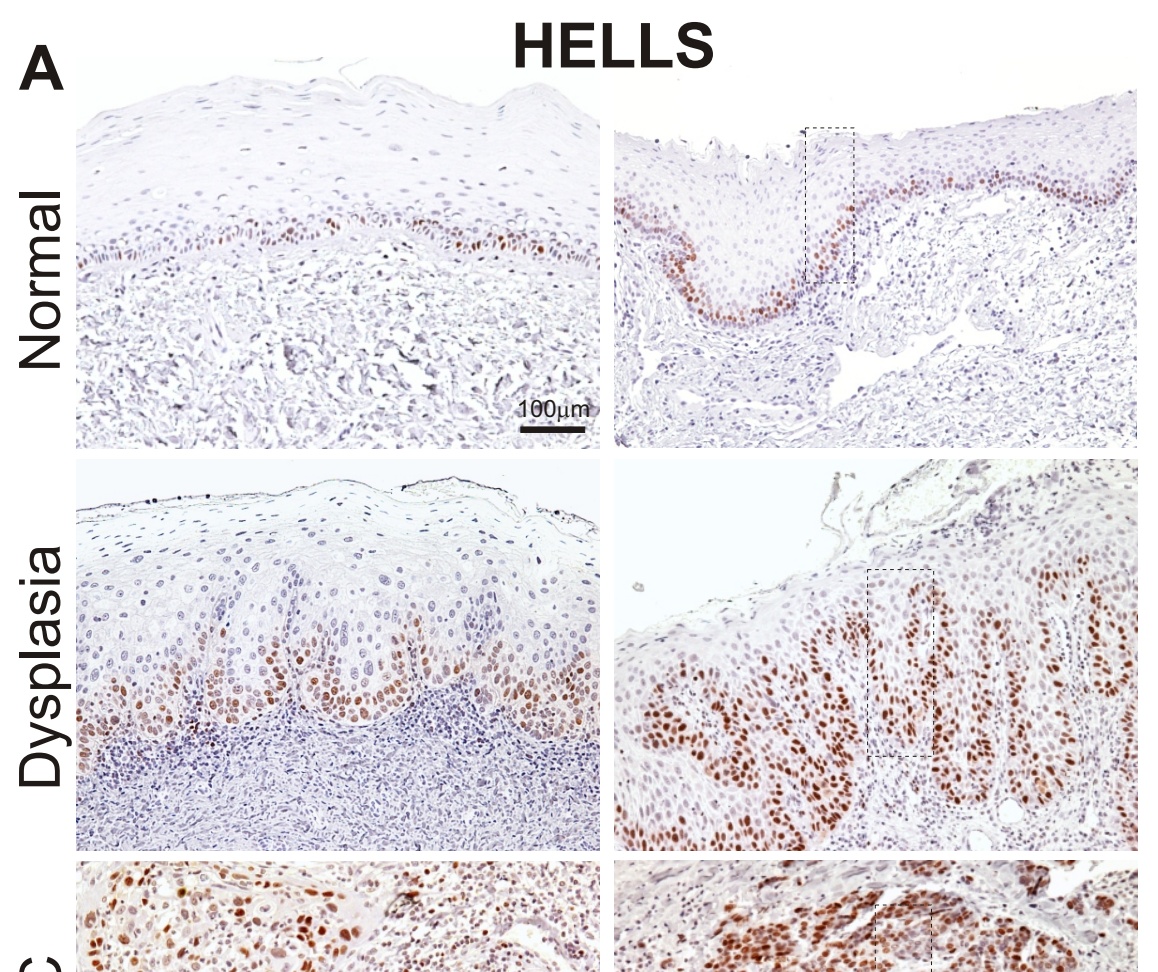

0
U
I
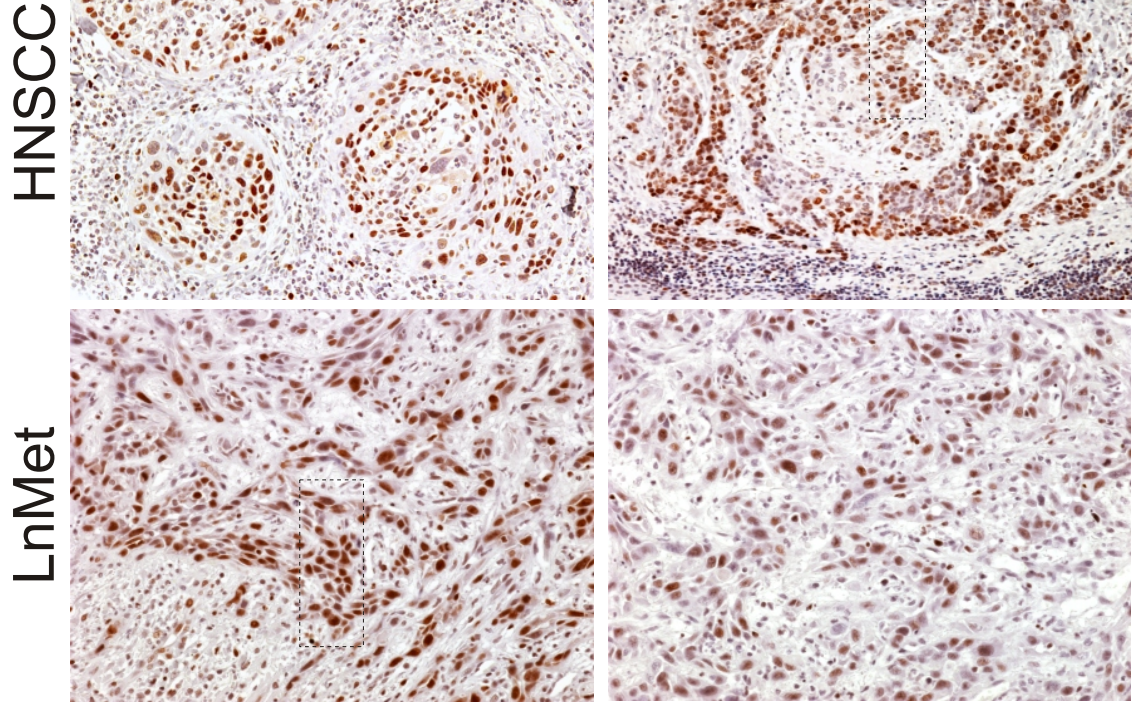

$\stackrel{+}{\Sigma}$

\section{B}
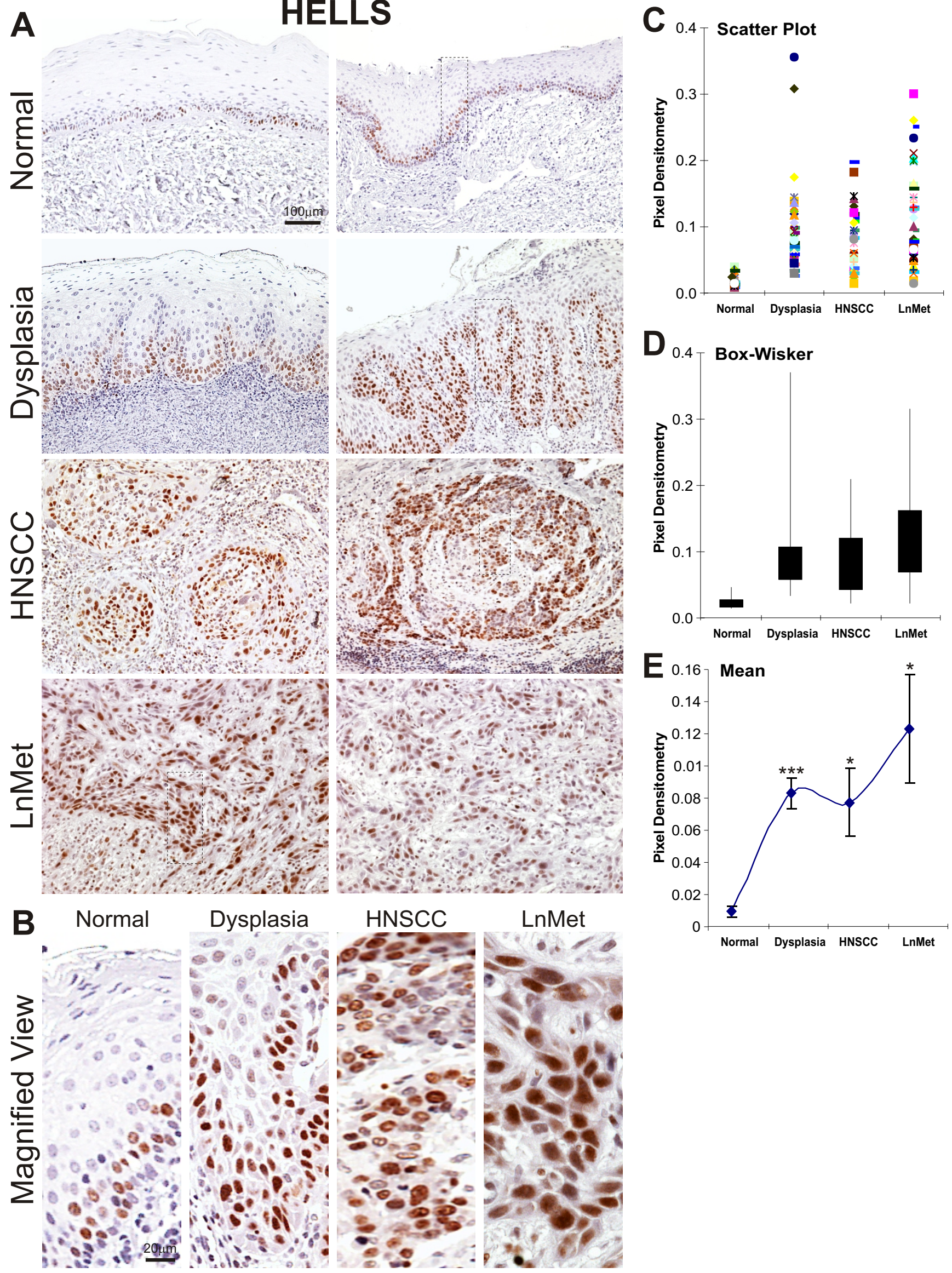
Figure 3

A

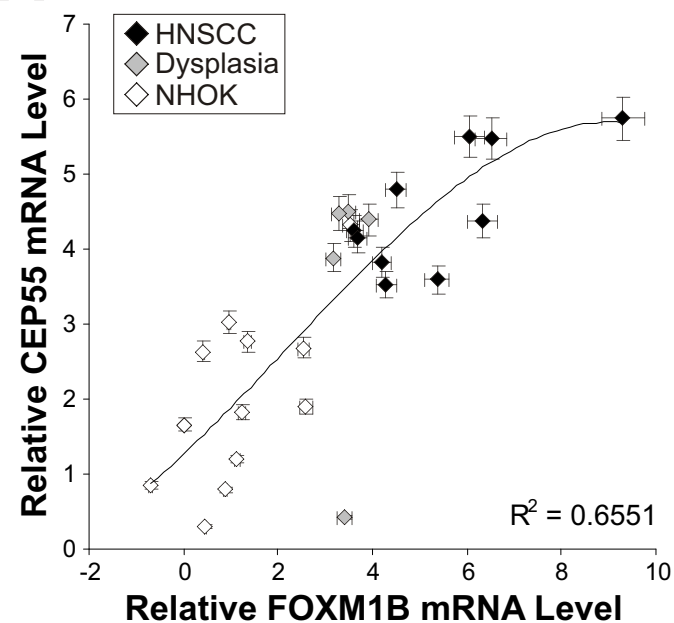

C
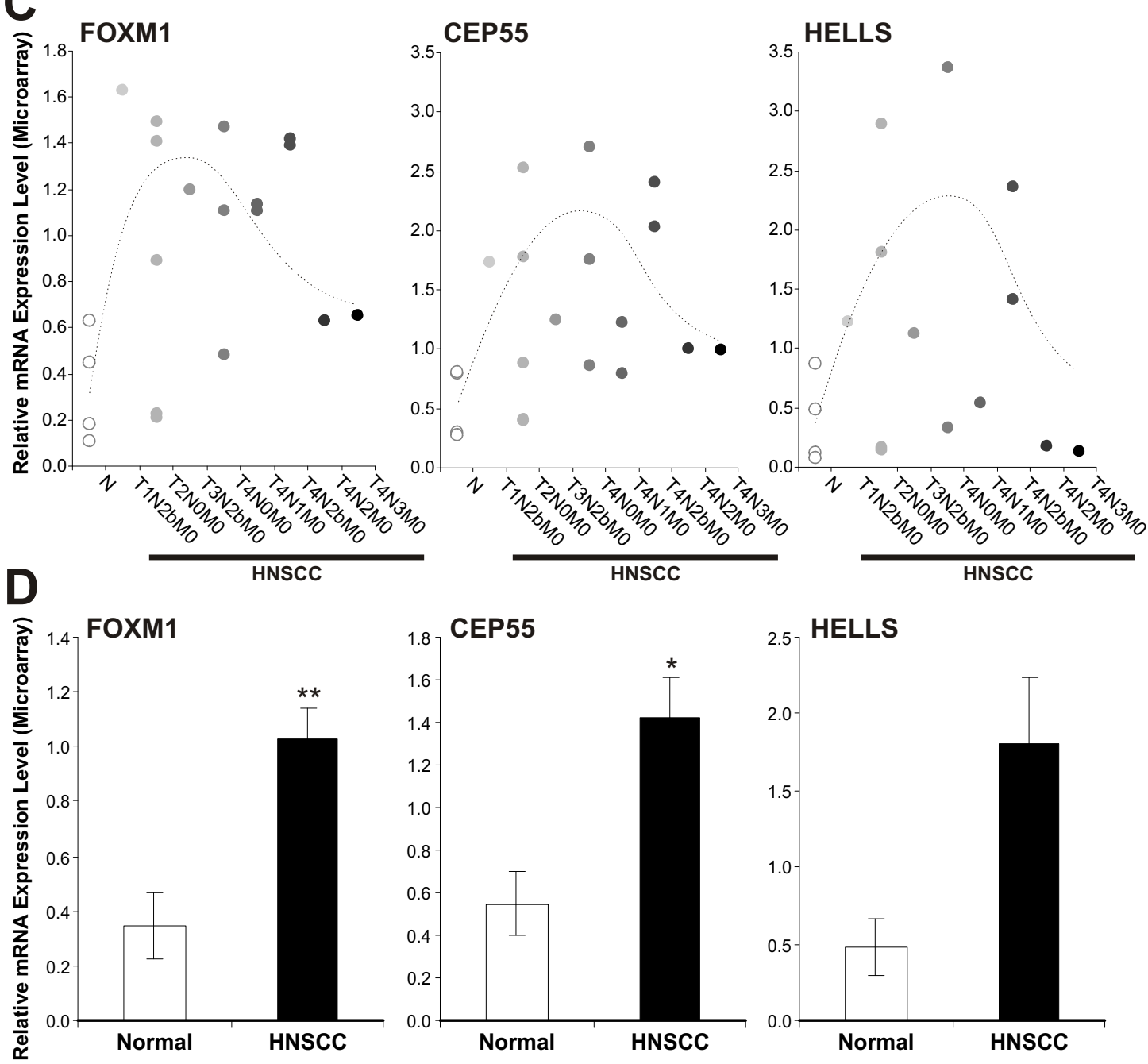